\title{
Finite Element Analysis of Asymmetric Scanning Near Field Optical Microscopy Probes
}

\author{
Valeria Lotito ${ }^{1,2, *}$, Urs Sennhauser ${ }^{1}$, and Christian Hafner ${ }^{2}$ \\ ${ }^{1}$ EMPA, Swiss Federal Laboratories for Materials Testing and Research, Electronics/Metrology Laboratory, \\ Ueberlandstrasse 129, $\mathrm{CH}-8600$ Dübendorf, Switzerland \\ ${ }^{2}$ Laboratory of Electromagnetic Fields and Microwave Electronics, ETH Zurich, Gloriastrasse 35, 8092 Zurich, Switzerland
}

\begin{abstract}
We investigate the effects of the introduction of new forms of asymmetries in a metal-coated scanning near field optical microscopy probe on the coupling of modes by numerical simulations. Because of the high computational efforts involved in such a study, the analysis is limited to the very end of the tip. Nevertheless some important trends in the overall probe behaviour are identified and different mechanisms to induce coupling between a tinearly polarized input mode and a radially polarized mode are detected. The latter mode is known to produce a strongly localized optical near field, a coveted feature for scanning near field optical microscopy applications.
\end{abstract}

Keywords: Scanning Near Field Optical Microscopy, Apertureless Optical Probes, Near Field Optics, Probe Geometry.

\section{INTRODUCTION}

Scanning near field optical microscopy (SNOM) has emerged as an important tool for the investigation of nanostructures over the past decades because it combines the potentiality of scanning probe technology with the power of optical microscopy. ${ }^{1-3}$ High resolution beyond the diffraction limit is reached by exploiting the nonpropagating evanescent waves in the near field zone, in order to obtain the high spatial frequency information of the object. In particular, according to whether the near field interactions are used to excite the sample and/or to collect the signal coming from the sample, basically three different SNOM operating modes can be distinguished. ${ }^{2}$ The so-called illumination mode is based on the near field excitation of the structure to be analyzed and on the far field detection of the propagating waves resulting from an interaction between the near field and the sample. Collection mode is given by just the reverse situation, which means that the sample is broadly irradiated with a far field excitation and the near field response is detected using a sub-wavelength optical probe. Use of near field interactions for both excitation and detection results in the illumination/collection mode, known also as double passage because light has to pass twice through the optical probe. The latter configuration is highly attractive because of its potential applications to non-transparent samples, but

\footnotetext{
*Author to whom correspondence should be addressed.
}

inherently requires much better throughput than the other two operating modes.

The choice of the probe plays a paramount role in SNOM applications because it determines the interaction with the analyzed sample. No matter whether the probe is used as a local illuminator, a local collector or both, the optical spatial resolution only depends on the confinement of the optical energy at the apex of the probe. ${ }^{2}$ This explains the search for a continuous optimization of the structure of such a key component.

The first probes to be proposed were based on metalcoated dielectrics with a transparent spot at the apex, called aperture probes. ${ }^{2,4,5}$ The most common example is a tapered optical fiber coated with metal, often aluminium. Such a probe can be viewed as a tapered hollow metal waveguide filled with a dielectric, along which different modes can propagate. The mode structure changes as a function of the characteristic dimension of the dielectric core. ${ }^{2,6}$ Linearly polarized $\mathrm{HE}_{11}$ modes are usually used for their excitation, resulting in asymmetric near field intensity distribution. ${ }^{7}$ Moreover, aperture probes are characterized by poor resolution, limited mainly by the aperture size, and low throughput. ${ }^{2}$

Apertureless metal-cladded SNOM probes have drawn much attention recently. Fully metal-coated SNOM probes are particularly interesting because of their high-volume manufacturability, the greater manufacturing reproducibility and the easier control over their shape. ${ }^{8}$ Moreover, cantilever-based apertureless microfabricated probes have 
been used in a hybrid SNOM/AFM set-up to perform optical as well as topographic measurements at the same time. Typically, they have been excited either by illumination from a focusing lens or in a prism-based total internal reflection configuration. ${ }^{7,9}$ In spite of the high resolution demonstrated, tips based on external illumination suffer from strong background; therefore, in an alternative approach, internal back excitation can be used. ${ }^{7,9,10}$

The polarization state of the input field is of critical importance for their resolution. The eigenmodes of the probe input aperture are given by a pair of orthogonal linearly polarized modes, followed by a radially polarized mode. Since higher order modes are strongly attenuated when propagating along thin structures, it is sufficient to focus on the linearly and radially polarized modes. As shown in both numerical and experimental studies, the radially polarized mode exhibits a localized hot spot in the near field zone of the probe apex together with a peak amplitude higher than the one of the linearly polarized modes. ${ }^{71-13}$ This feature is clearly desirable for high resolution microscopy applications. As a consequence, the internal excitation of the radially polarized mode has been thoroughly scrutinized both analytically ${ }^{9,14}$ and numerically. ${ }^{10-12,15-17}$ The strong localization achievable with a radially polarized excitation has been explained as the outcome of the energy transfer from the waveguide mode (WGM) to the surface plasmon polariton (SPP) occurring when the wave vectors of the WGM and the SPP are equal. ${ }^{14}$ The induced surface plasmon excited on the metal surface will converge towards the end of the tip and interfere constructively due to the rotational symmetry of the input polarization and the probe. It is important to note that if, on the other hand, a linearly polarized beam is coupled into a purely axisymmetric probe, the surface plasmon excitation will cancel out because of the opposite charges on the opposite sides of the tip. ${ }^{16,17}$ In general, no mode conversion is possible on an axisymmetric structure. For telecommunication applications, this is often desirable.

The polarization-dependent resolution characteristics of apertureless probes have stimulated interesting studies about how to induce a conversion mechanism from a more easily excitable linearly polarized mode to the radial one, which provides better resolution. In fact, even though the selective control of the injection of focused linearly and radially polarized beams into cantilevered microfabricated SNOM probes has been demonstrated, this is far from being a straightforward task. In particular, the injection of a focused radially polarized beam is highly sensitive to misalignments. For example, referring to the injection method reported in Ref. [13], the most delicate step consists in bringing the optical axis of the focusing system as close as possible to the probe axis: if the focused beam does not have a fully circular symmetry at injection, the dominant linear field component will couple to other modes in a non controlled way. As apparent, this would clearly impair the potential benefits stemming from the use of radially polarized modes.

One of the possible routes to achieve mode coupling is based on the introduction of geometric modifications breaking the axial symmetry of the standard tip (Fig. 1(a)). One study about conversion mechanisms dealt with the analysis of the influence of defects in the metal coating onto the mode coupling. ${ }^{8}$ Different defects shapes, sizes and configurations were examined and it was shown that particular defect placements could induce mode coupling. Given $z$ the direction of the axis of the probe, the effect of the introduction of single (Fig. 1(b)) and multiple spherical defects, i.e., bubbles, in the metal layer on the $x-z$ plane was investigated. It was shown that, apart from differences related to the size, the number and the longitudinal position of the bubbles, such defects induced the coupling of one of the two linearly polarized modes into the radially polarized one, the other orthogonal linearly polarized mode being almost unaffected. If another bubble was added in the orthogonal $y-z$ plane (Fig. 1(c)), both linearly polarized modes could be converted into the radially polarized one, while two identical bubbles at diametrically opposite locations (Fig. 1(d)) resulted in a net zero coupling to the radial mode. Therefore, destructive interference or linear addition of the scattering contributions of the single defects can ensue from certain specific configurations, resulting in no coupling, coupling of one of the two linearly polarized modes or coupling of both of them. As real probes have a large number of random defects and the overall behaviour of the probe is governed by the aggregate behaviour of such defects, a coupling based on such a mechanism is not easily predictable. However specific intentional defects could produce the desired conversion. Numerical studies about unilateral (Fig. 1(e)) and bilateral slits (Fig. 1(f)) have demonstrated their ability to induce coupling of one of the two linearly polarized modes into the radially polarized one. ${ }^{18}$ The problem of the background due to leakage of the other orthogonal linearly polarized mode seemed to be significantly less important in the case of a bilateral slit. Moreover the unilateral slit requires a very well defined and oriented input linear polarization state.

In this work, we first examine the effect of another type of intentional asymmetry based on an oblique cut on the coupling properties of the probe. Compared to the asymmetric structures considered in previous studies, the proposed configuration is no longer properly apertureless as the cut involves also the dielectric core of the metal cladded tapered structures. However, phenomena similar to those highlighted in the aforementioned studies can still be observed. This form of asymmetry is easier to be realized in a real fabrication process. Then, benefitting from the aggregate cumulative behaviour of asymmetries, we analyze the effect of the combination of asymmetries located in orthogonal directions on the performance of the probe. Two 
$\times 1 e^{-7}$

(a)

(a)

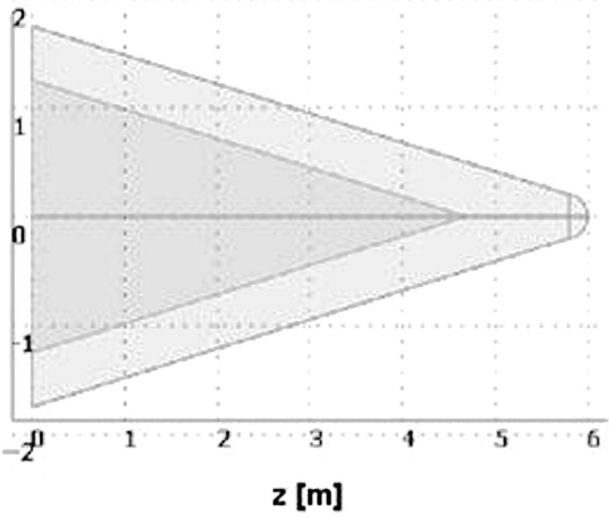

$\times 1 e^{-7}$

(c)

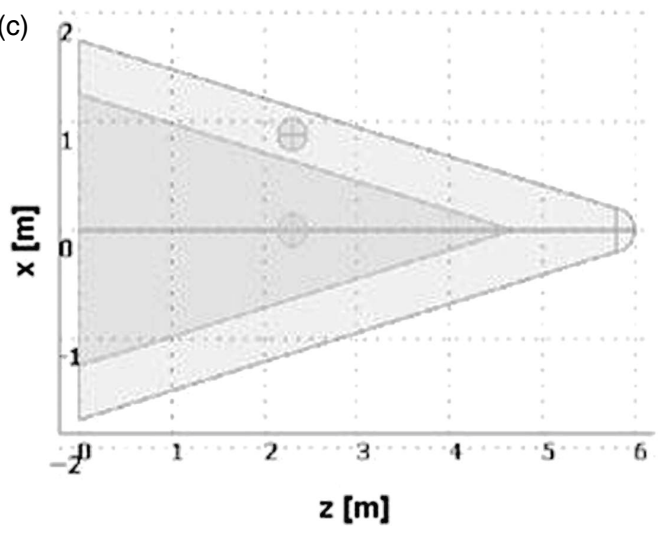

$\times 1 e^{-7}$

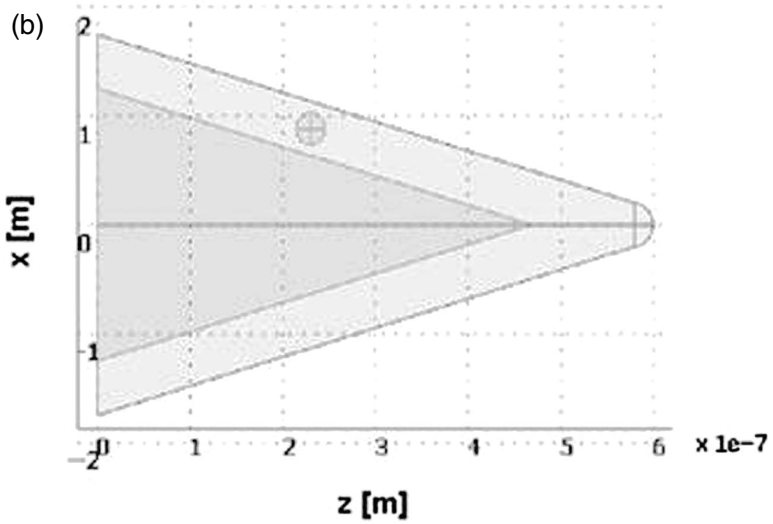

$\times 1 e-7$

(d)

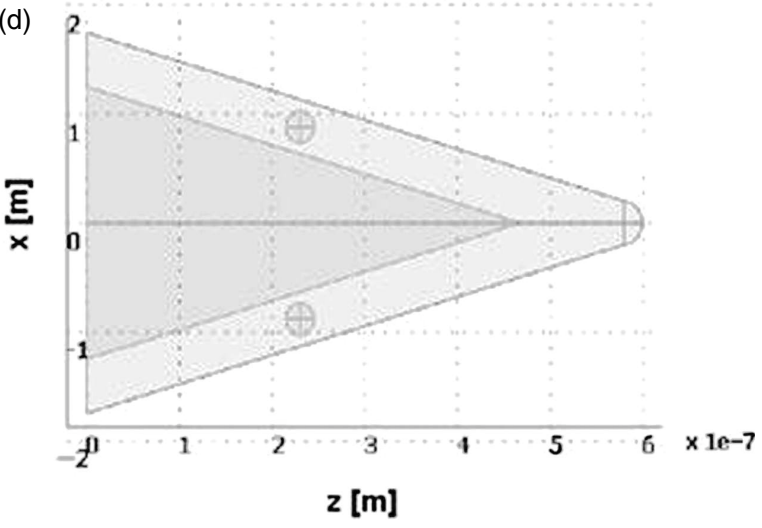

(e)

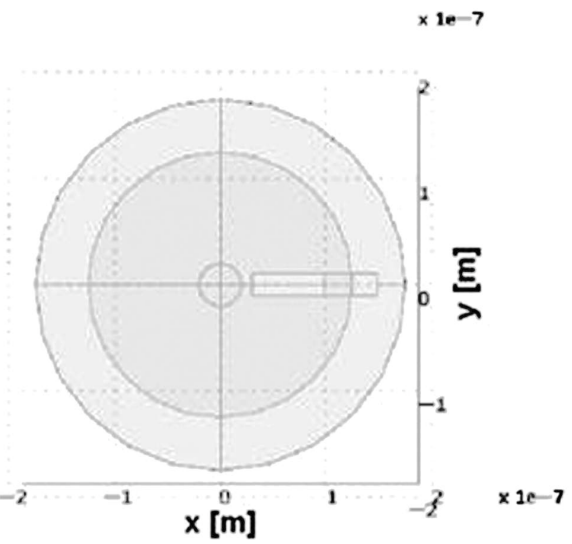

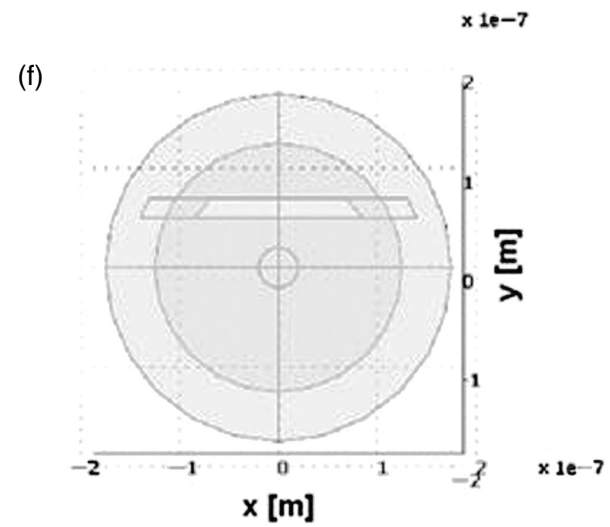

Fig. 1. Examples of asymmetries introduced in axisymmetric probes: (a) standard fully metal coated axisymmetric probe (projection on $z-x$ plane); (b) single defect in the metal layer (projection on $z-x$ plane); (c) two defects in the metal layer located in orthogonal planes (projection on $z-x$ plane); (d) two defects in the metal layer located at diametrically opposite positions (projection on $z-x$ plane); (e) unilateral slit (projection on $x-y$ plane); (f) bilateral slit (projection on $x-y$ plane).

possible configurations likely to induce coupling of both linearly polarized modes into the radially polarized one are detected. Their adoption could potentially reduce the criticality of input polarization injection and add to the overall resolution of the probe. The effect of different geometrical parameters on the near field distribution is investigated. Due to computational limitations, only the very end of the tip will be examined. Nonetheless, a satisfactory qualitative description of the mode coupling is provided for these promising geometrical configurations of the probe.

Different numerical methods have been used in the past to simulate fully metal coated SNOM tips, ranging from multiple multipole method ${ }^{10,17}$ to the finite integration time domain technique $8,9,11,13,18$ or the finite difference 


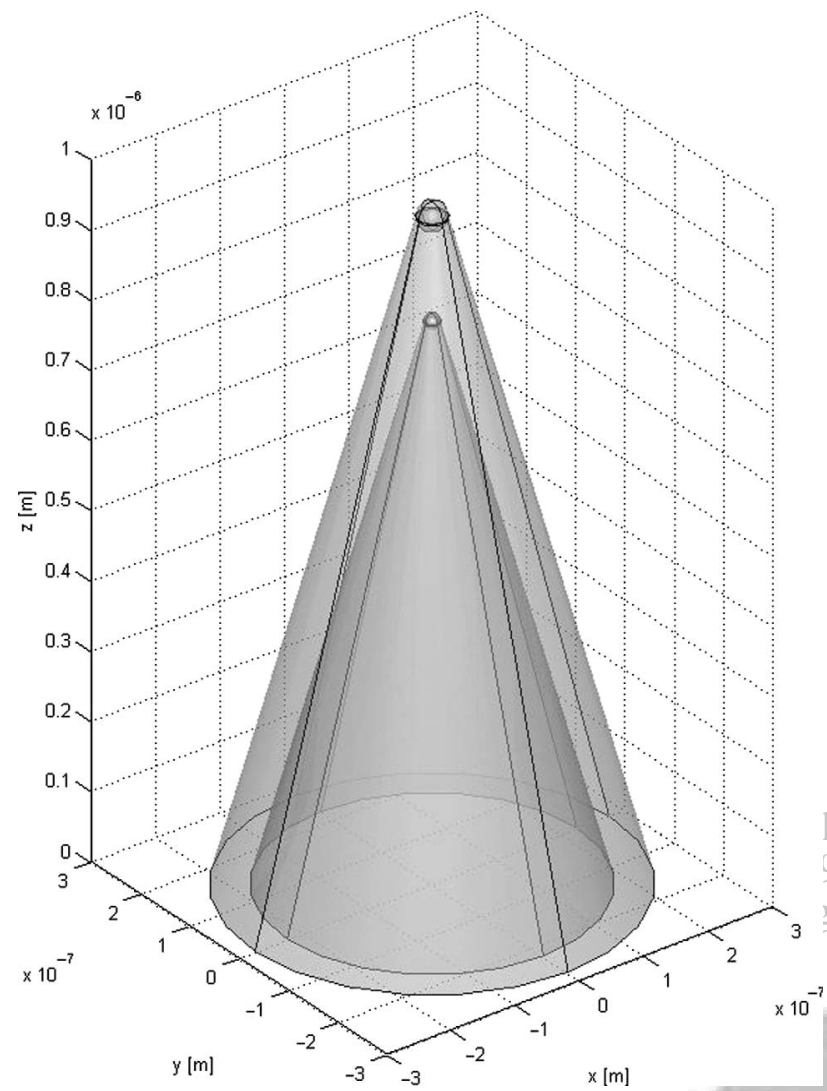

Fig. 2. Standard fully metal-coated probe.

time domain method. ${ }^{7}$ Finite element method, used also in Refs. [15, 16], was chosen to carry out our simulations because of the ease in handling complicated geometries as the ones considered in this paper. The use of unstructured grids can accommodate for irregular shapes in a much more straightforward way than structured grids.

\section{COMPUTATIONAL MODEL}

The three-dimensional (3D) computational model for the simulation of the electromagnetic modes in the investigated probe configurations has been developed with the help of a commercial software (Comsol Multiphysics) based on the finite element method. The computational process consists of two steps. First a two-dimensional (2D) analysis is run to calculate the eigenmodes at the input port. Then, the first three eigenmodes, i.e., the two lowest order linearly polarized modes and the radially polarized mode, are propagated through the probe in a 3D simulation.

The structure of the standard fully metal coated axisymmetric probe is reported in Figure 2. The overall modelling domain is a cylinder with radius $1 \mu \mathrm{m}$ and height $1.6 \mu \mathrm{m}$. The probe axis is chosen to lie on the $z$ axis. The probe itself consists of a cone of silica core $(n=1.5)$ surrounded by an aluminium coating $(n=0.645+5.029 i$ at the operating wavelength $\lambda=532 \mathrm{~nm}$ ). The radius of the inner silica cone is $225 \mathrm{~nm}$, while the radius of the metallic hollow cone is $275 \mathrm{~nm}$. The apex angle for both cones is $30^{\circ}$. Both cones are rounded: the radius of curvature of the inner cone is $10 \mathrm{~nm}$, the one of the outer cone is $20 \mathrm{~nm}$.

The first three eigenmodes are reported in Figure 3. The mode labelled as $\mathrm{H}$ is a primarily linearly polarized mode with a dominant $E$ field vector parallel to the $x$ axis; the one denoted by $\mathrm{V}$ is the orthogonal linearly polarized mode with a dominant $E$ field vector parallel to the $y$ axis. Finally we have the radially polarized mode called R, responsible for the hot spot in the near field.

In the subsequent sections, each of these modes will be taken as input port for the 3D simulations in the different structures. First, an evaluation of the emitted near field near the apex of the probe will be done for the standard axisymmetric probe that will act as reference probe for the other configurations. Next, the new asymmetry consisting of an oblique cut will be analyzed. The following step will be that of combining asymmetries in orthogonal directions to induce the coupling of the $\mathrm{H}$ and $\mathrm{V}$ modes into the $\mathrm{R}$ mode: the analyzed configurations will result from the combination of either two orthogonal slits or the ensemble of a slit and an oblique cut in the orthogonal direction.
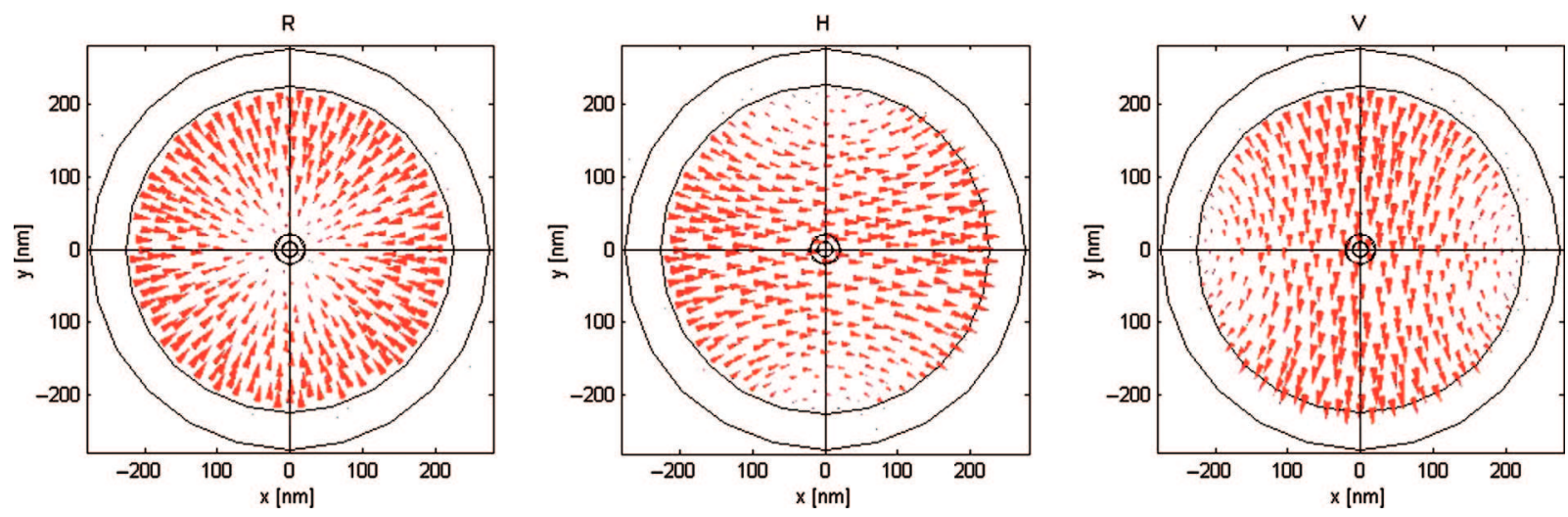

Fig. 3. The three polarization eigenmodes: the radially polarized mode $\mathrm{R}$ and the two orthogonal linearly polarized modes $\mathrm{H}$ and $\mathrm{V}$. 


\section{STANDARD FULLY METAL COATED PROBE}

In order to have a reference to compare the other modified structures with, a simple fully metal coated probe has been simulated. The field distribution in a transverse plane placed at $10 \mathrm{~nm}$ from the tip apex has been examined for each of the three modes. The square of the norm of the electric field for the three cases is reported in Figure 4. The plots are taken over a square area $600 \mathrm{~nm}$ by $600 \mathrm{~nm}$ centered at the origin of the coordinate system and are normalized to the maximum value of the electric field intensity distribution for each of the modes in order to emphasize the details of each field distribution. Unless otherwise stated, such a plane will be considered for the representation of the normalized field distributions also in the following paragraphs. As visible, the $\mathrm{R}$ polarized mode gives rise to a strongly localized hot spot, originating from the composition of two weaker two-lobed transversely polarized patterns and a stronger single spot $z$-component. As mentioned above, the formation of this ultra-small hot spot has been explained as resulting from the excitation of surface waves propagating along the taper and interfering constructively at the tip apex. The full width at half maximum (FWHM) of the intensity of the electric field has been calculated to be $38 \mathrm{~nm}$. On the other hand, the $\mathrm{H}$ and $\mathrm{V}$ polarized modes give rise to a field distribution that is characterized by the presence of two lobes and is mainly $x$ and $y$ polarized respectively. In this case the fields are extended over a much broader area. The shape of the intensity distribution is not well defined but the average distance over which the field is more than or equal to half of its peak value is approximately $400 \mathrm{~nm}$ for both linearly polarized modes. The peak value of the $\mathrm{R}$ hot spot is about 50 times higher than the peak of the two linearly polarized modes.

As observed in Ref. [8], there are many significant features of the near field distributions that could be taken

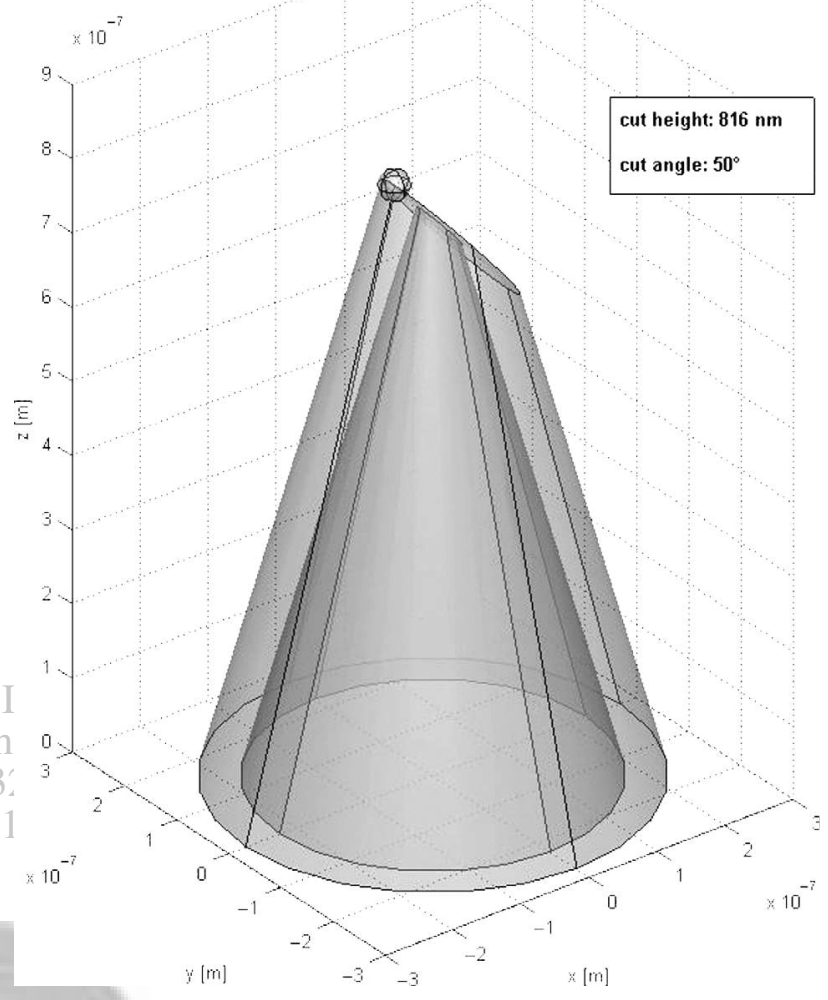

Fig. 5. Cut probe (cut height $816 \mathrm{~nm}$; cut angle $50^{\circ}$ ).

into account to characterize the optical behaviour of the probe and to have meaningful guidelines for comparison. Besides a qualitative description of the overall shape of the field and the size of the emitted spot, characteristics like peak field amplitude and ratio of the peak to the background amplitude have been indicated as useful traits. If needed, some additional parameters useful to better characterize the particular structure at hand will be introduced.

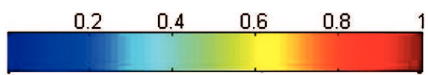

$\mathbf{R}$

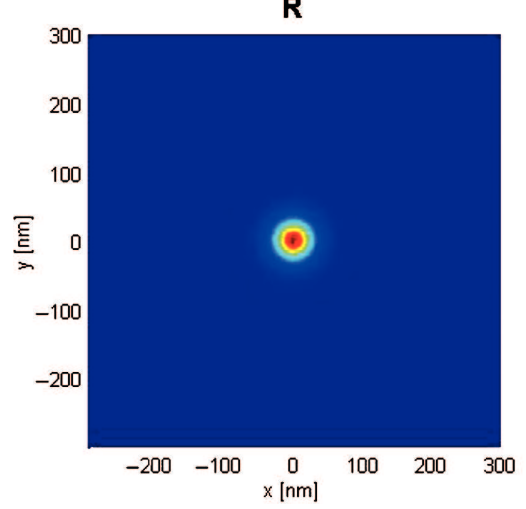

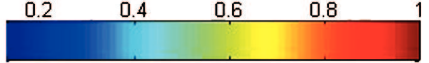

H

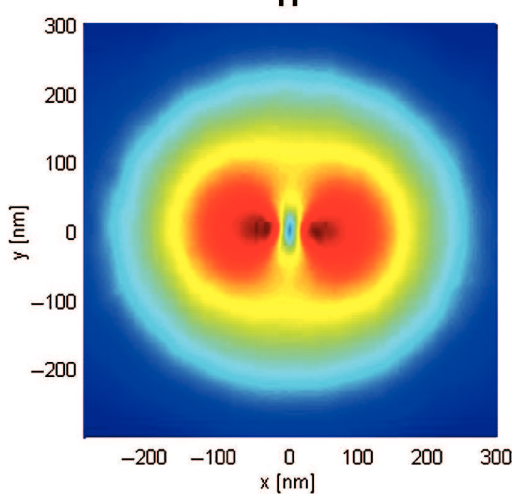

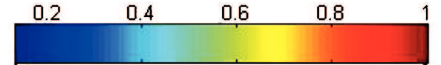

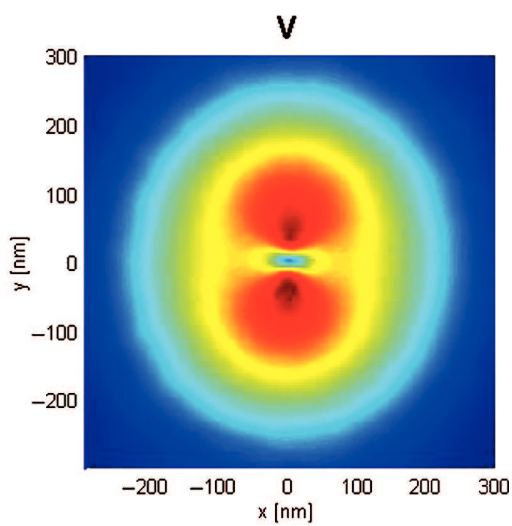

Fig. 4. Normalized electric field intensity at $10 \mathrm{~nm}$ from the apex for the axisymmetric fully metal-coated probe. 


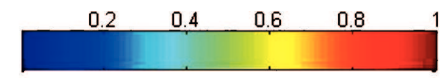

$\mathbf{R}$

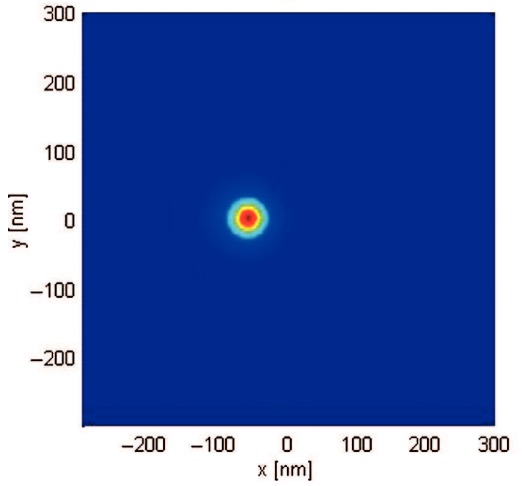

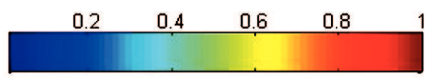

H

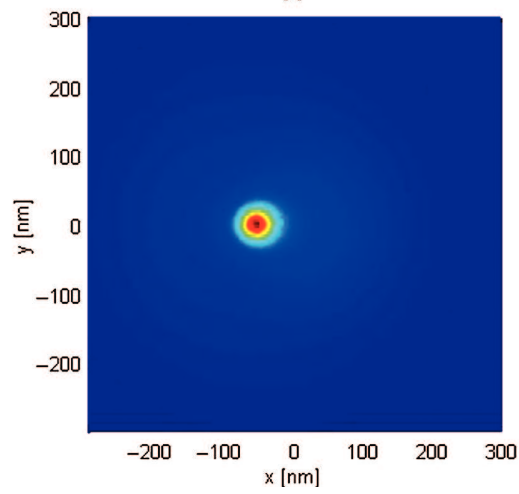

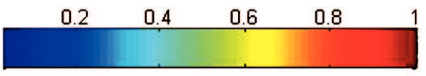

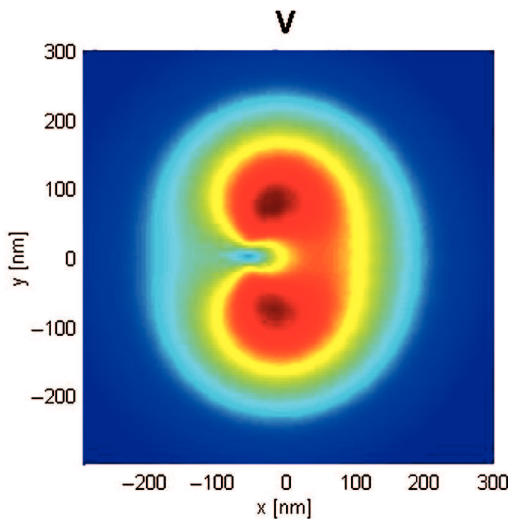

Fig. 6. Normalized electric field intensity at $10 \mathrm{~nm}$ from the apex for the cut probe shown in Figure 5 .

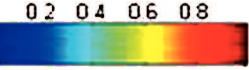

$\mathbf{R}$
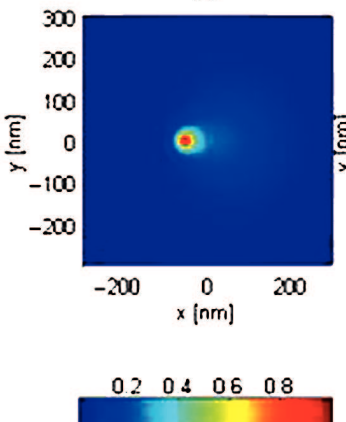

H
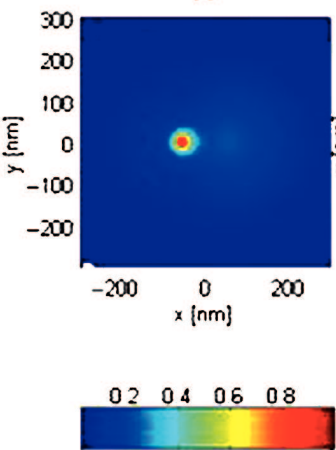

V

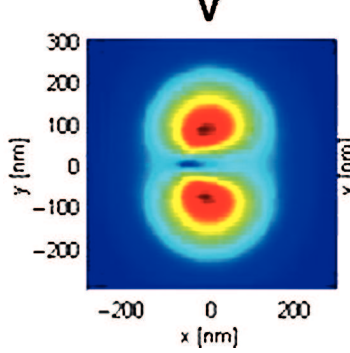

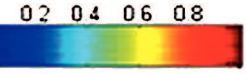

$\mathbf{R}$
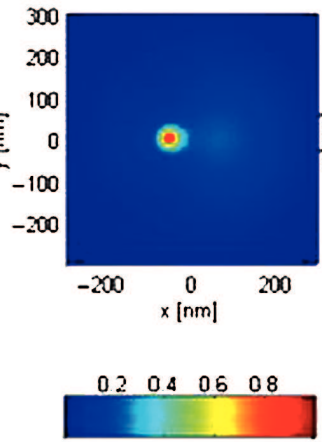

H
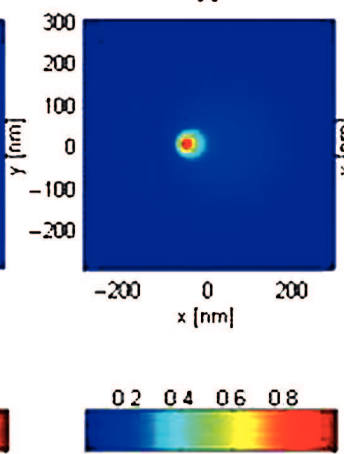

V

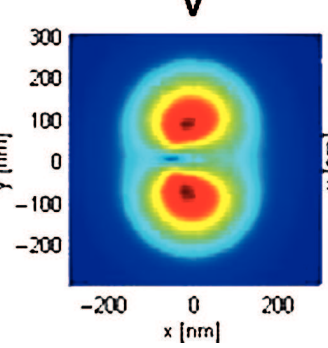

$020406 \quad 08$

$\mathbf{R}$
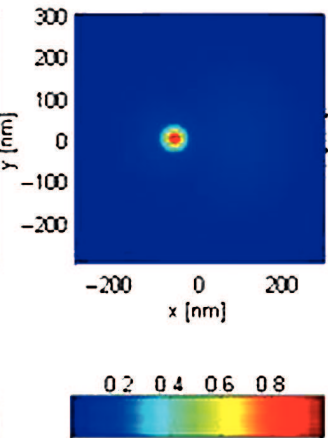

H
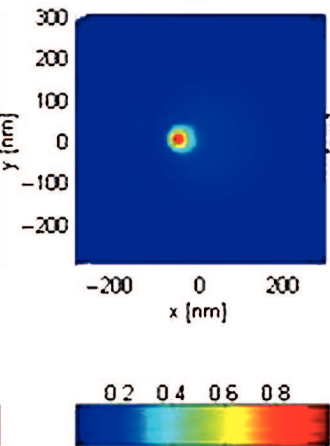

v

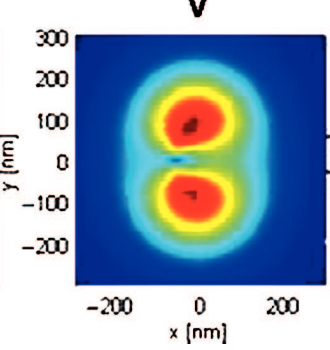

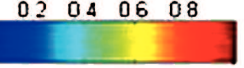

$\mathbf{R}$
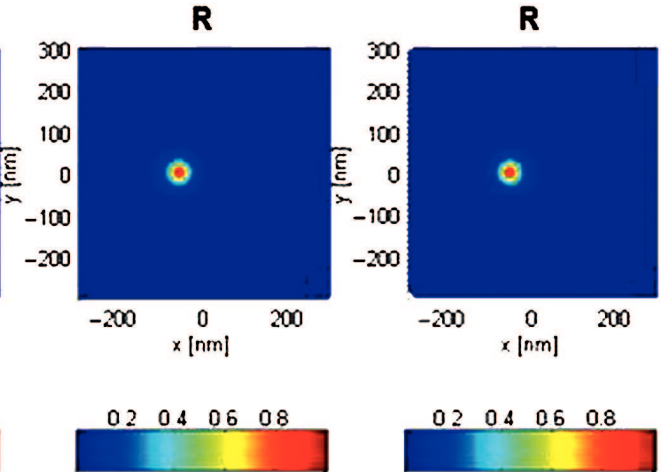

H
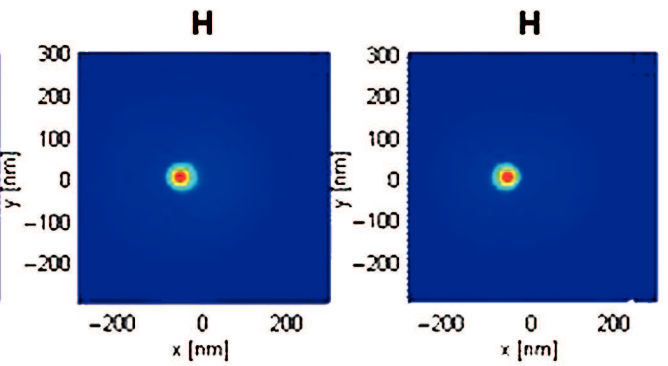
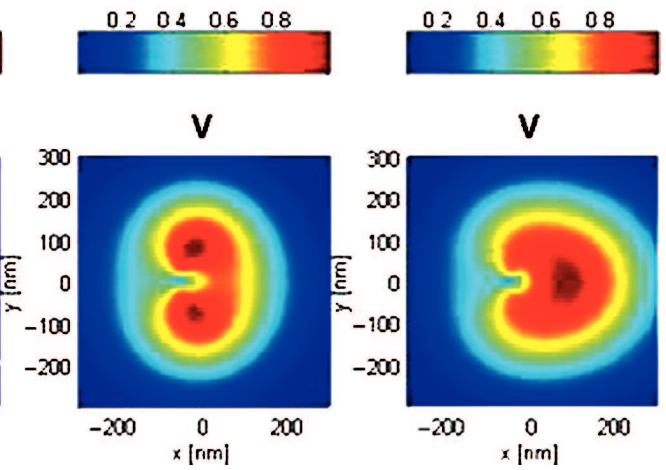

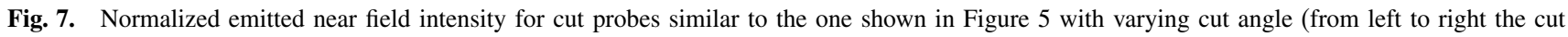
angle changes from $20^{\circ}$ to $60^{\circ}$ with a step of $10^{\circ}$; the cut height is set to $816 \mathrm{~nm}$ ). 

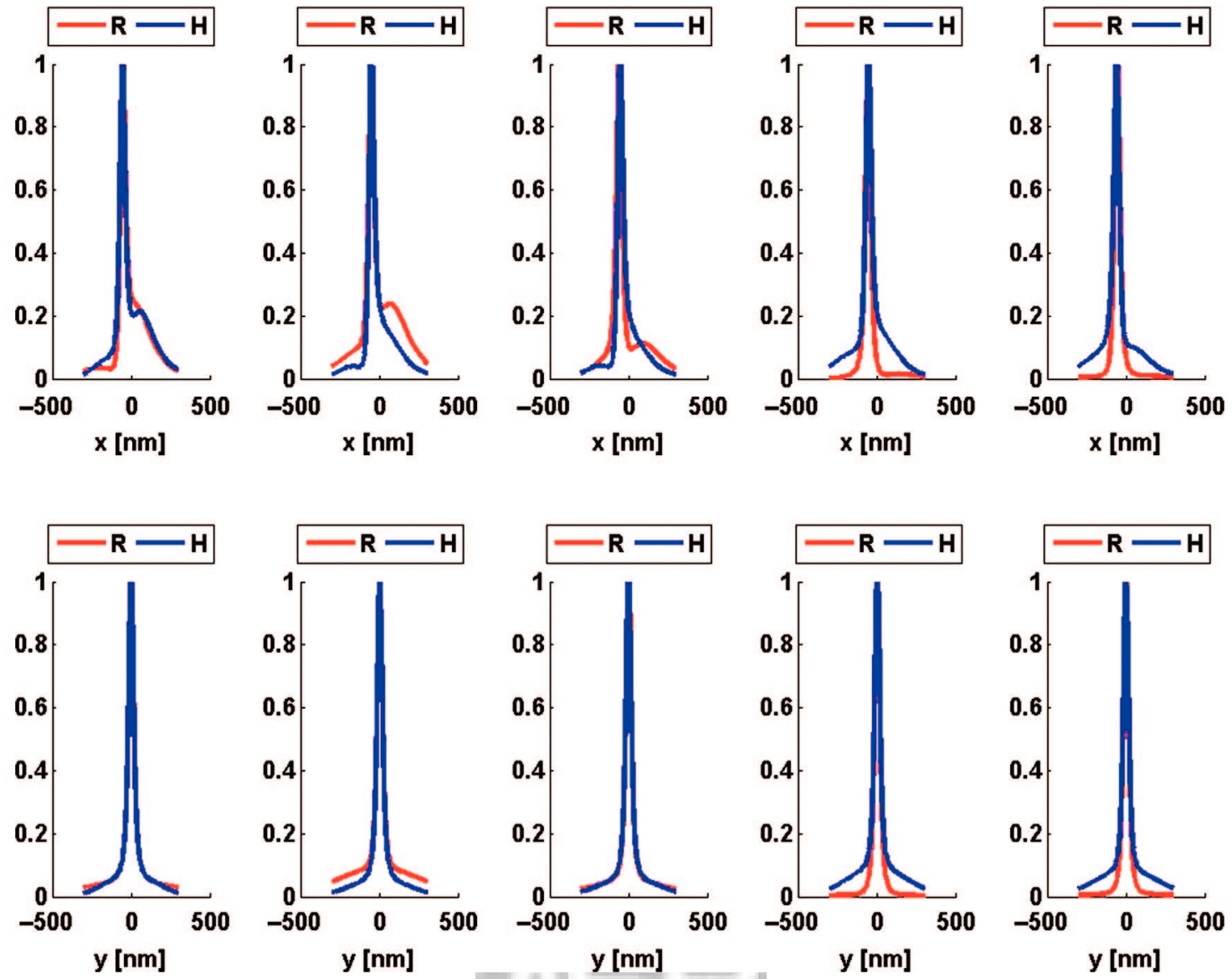

Fig. 8. Normalized cross section profiles for cut probes similar to the one shown in Figure 5 at different cut angles (from left to right the cut angle changes from $20^{\circ}$ to $60^{\circ}$ with a step of $10^{\circ}$; the cut height is set to $816 \mathrm{~nm}$ ).

\section{SINGLE CUT TIP}

A new kind of asymmetry has been considered, consisting in an oblique cut stripping off the top of the probe and intersecting both the metal coating and the core. A picture of the resulting tip is shown in Figure 5. The height of the uppermost point of the cut is $816 \mathrm{~nm}$ and the angle of the cut defined between a transverse plane orthogonal to the $z$ direction and the plane of the cut itself is equal to $50^{\circ}$. A sphere of radius equal to $20 \mathrm{~nm}$

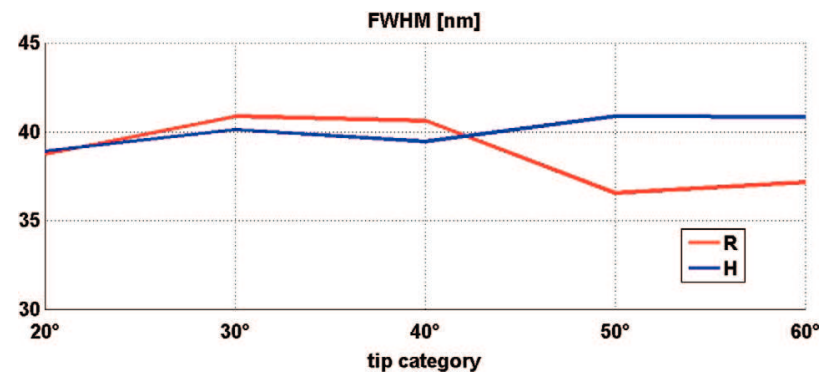

Fig. 9. FWHM for cut probes similar to the one shown in Figure 5 at different cut angles (from left to right the cut angle changes from $20^{\circ}$ to $60^{\circ}$ with a step of $10^{\circ}$; the cut height is set to $816 \mathrm{~nm}$ ). centered at the new tip apex resulting from the cut has been introduced.

The normalized electric field intensity collected at the output plane is reported in Figure 6.

As the asymmetry is along the $x$ axis, the $\mathrm{H}$ mode output field distribution undergoes shrinkage, while the $\mathrm{V}$ mode in not substantially affected. The FWHM for the $\mathrm{H}$ mode is about $41 \mathrm{~nm}$, that of the $\mathrm{R}$ electric field distribution is roughly $37 \mathrm{~nm}$. While the R mode keeps on having an axial symmetric distribution about its peak, the

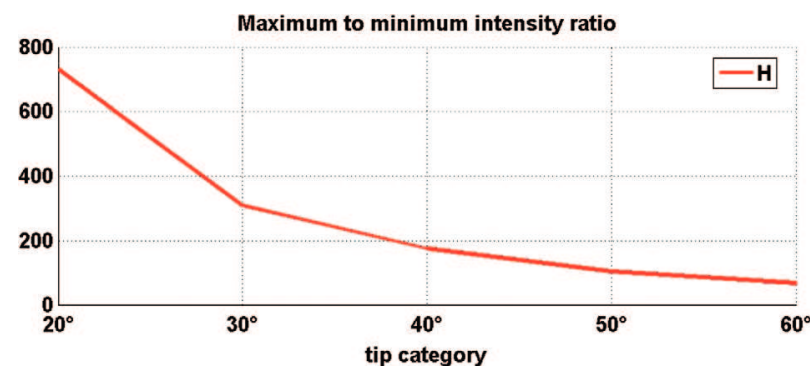

Fig. 10. Contrast for cut probes similar to the one shown in Figure 5 at different cut angles (from left to right the cut angle changes from $20^{\circ}$ to $60^{\circ}$ with a step of $10^{\circ}$; the cut height is set to $816 \mathrm{~nm}$ ). 
$\mathrm{H}$ mode appears to be asymmetric along the $y$ axis and shifts preferentially towards the cut area. As will be shown later, the asymmetry can be reduced by properly tuning some geometric parameters for the cut probe configuration. However, it is worth to remark that, even in this case, the intensity components of the hot spot above one half of the peak value preserve almost a circular shape in the $x-y$ plane. All these features will be further investigated in the following paragraphs.

\section{PARAMETRIC STUDY OF THE SINGLE CUT CONFIGURATION}

As mentioned above, the effect of the change in some geometric parameters has been evaluated for the single cut configuration. First of all, the angle of the cut has been changed from $20^{\circ}$ to $60^{\circ}$ with a step of $10^{\circ}$, keeping the cut apex at $816 \mathrm{~nm}$. Results in the output near field distribution are shown in Figure 7.

For all the angles, a localized hot spot is obtained for both the $\mathrm{R}$ and $\mathrm{H}$ modes, as expected. The two lobes of the $\mathrm{V}$ mode that are initially separated tend to merge as the angle becomes steeper until getting completely intermingled, resulting in the formation of a strong spread in the field distribution in the cut area. As to the $\mathrm{R}$ and $\mathrm{H}$ spots, the initially asymmetric field distributions become progressively more symmetric about their center along the $x$ axis as the cut angle increases. However their FWHM does not change remarkably with the cut angle, as will be illustrated later.

To get more insight into the obtained field distributions, the normalized line profiles for the $\mathrm{R}$ and $\mathrm{H}$ modes along $x$ and $y$ directions have been plotted in Figure 8. For each of the two distributions, the profiles are taken along $x=x_{\max }$ and $y=y_{\max }$ where $\left(x_{\max }, y_{\max }\right)$ are the coordinates of the peak.

No strong variations occur in the profiles taken along $y$ : both $\mathrm{R}$ and $\mathrm{H}$ mode show good symmetry about the origin, with the $\mathrm{R}$ mode exhibiting a more rapid decay than the $\mathrm{H}$ mode as the angle increases. On the contrary, the mode profile along $x$ is quite asymmetric. However the initial strong asymmetry in the $\mathrm{R}$ and $\mathrm{H}$ modes is significantly reduced as the cut angle increases.

Figure 9 reports the FWHM for the different sets of simulations; as anticipated, no dramatic changes occur in the size of the spots which is always in the interval between $37 \mathrm{~nm}$ and $41 \mathrm{~nm}$.

In order to better characterize the optical resolution, aside from the graphs showing the decay with lateral displacement, the signal to background ratio for the $\mathrm{H}$ mode has been calculated over the area of the output plane (Fig. 10). As one can see, the contrast decreases as the angle increases. Nonetheless, it is still about 100 even for the steepest angle.

The ratio of the peak value of the $\mathrm{H}$ intensity distribution to that of the $\mathrm{R}$ mode for the standard fully metal-coated tip and that between $\mathrm{H}$ and $\mathrm{V}$ modes have been calculated, as shown in Figure 11.

Steeper cut angles result in an increase in the $\mathrm{H}$ peak with respect to the peak one would get using a fully metal coated axisymmetric tip excited with a radial beam. For very low angles, the intensity of the localized $\mathrm{H}$ spot is still very low, but, as the angle increases, it can reach values twice as the peak for the hot spot of the radial polarization. The ratio with respect to the peak of the $\mathrm{V}$ field distribution improves as well: the peak of the $\mathrm{V}$ distribution becomes a decreasing fraction of the $\mathrm{H}$ peak. As it is important to get a clearly detectable $\mathrm{H}$ hot spot, the results of the simulations suggest the use of steeper cut angles.

A similar analysis has been carried out to assess the impact of a variation in the cut height. The height was increased from $741 \mathrm{~nm}$ to $841 \mathrm{~nm}$ with a step of $25 \mathrm{~nm}$, while keeping the angle constant at $20^{\circ}$. Results for the output near:field are shown in Figure 12.

Also in this case, a localized spot is created for both $\mathrm{R}$ and $\mathrm{H}$ modes. The lobes of the $\mathrm{V}$ distribution tend to separate as the height increases. A study of the profile distributions along the $x$ and $y$ directions (Fig. 13) reveals again a good symmetry along the $y$ direction and an asymmetry along the $x$ direction which is particularly striking for the $\mathrm{H}$ distribution for higher cuts.

In this case the deterioration in the $\mathrm{H}$ field distribution is confirmed by the observed values of FWHM (Fig. 14). While the size of the $\mathrm{R}$ hot spot is not much affected by the cut height, the $\mathrm{H}$ spot swiftly increases as the cut becomes higher reaching values above $50 \mathrm{~nm}$ for the last set of simulations.
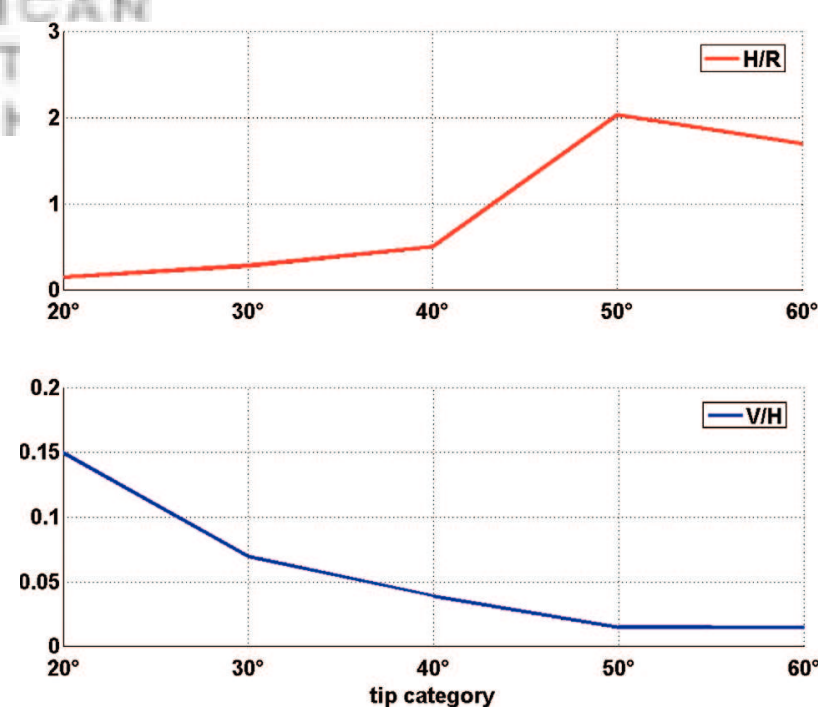

Fig. 11. Ratio between maxima for cut probes similar to the one shown in Figure 5 at different cut angles: the ratio with respect to the $\mathrm{R}$ mode is referred to the radial mode of the fully metal-coated tip (from left to right the cut angle changes from $20^{\circ}$ to $60^{\circ}$ with a step of $10^{\circ}$; the cut height is set to $816 \mathrm{~nm}$ ). 

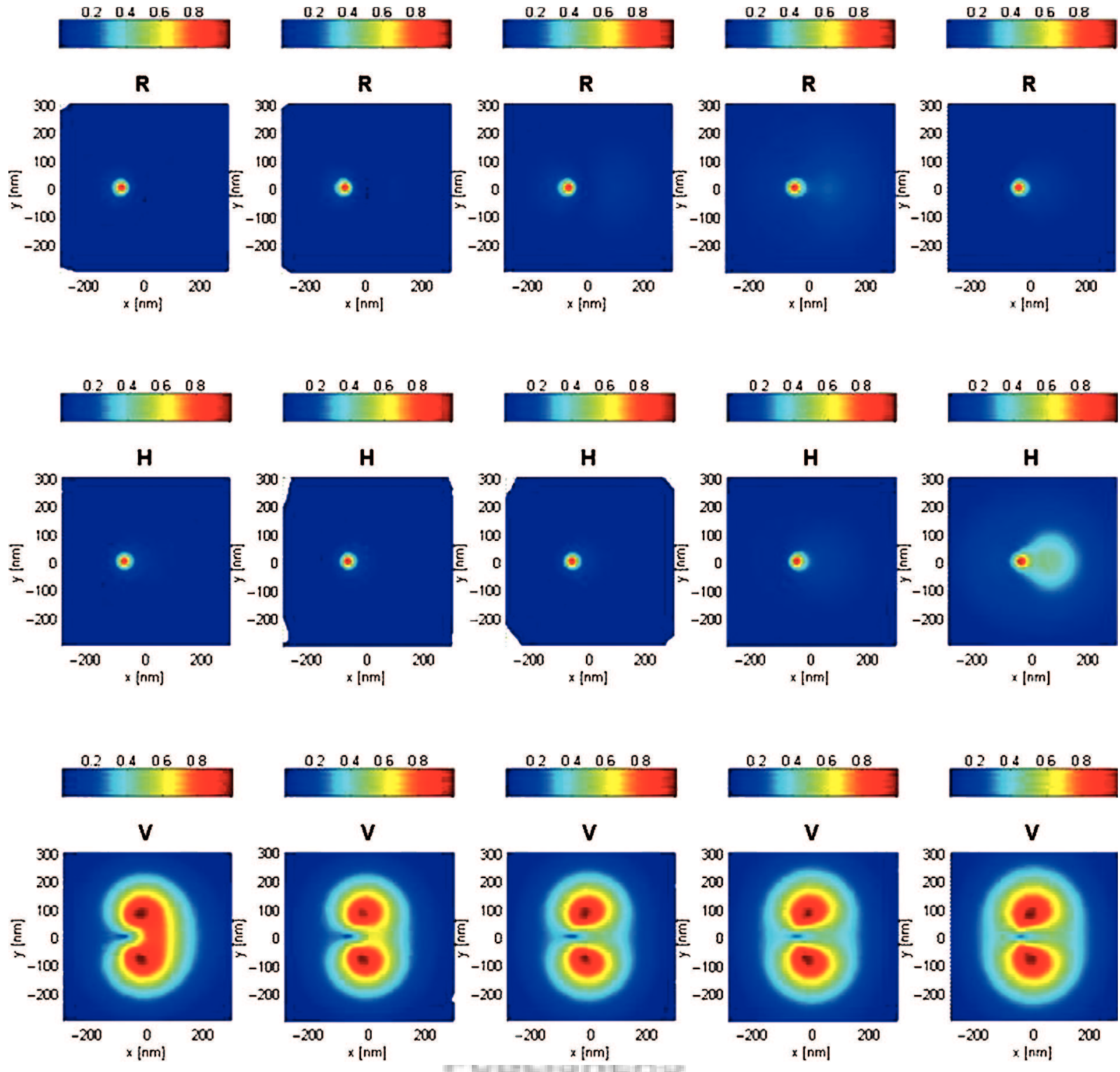

Fig. 12. Normalized emitted near field intensity for cut probes similar to the one shown in Figure 5 with varying cut height (from left to right the cut height is increased from $741 \mathrm{~nm}$ to $841 \mathrm{~nm}$ with a step of $25 \mathrm{~nm}$; the cut angle is set to $20^{\circ}$ ).

Moreover, also the contrast for the $\mathrm{H}$ mode decreases for increasing heights as reported in Figure 15, even if it is above 100 even in the last case.

The parameter that undergoes the worst degradation is the peak value of the $\mathrm{H}$ distribution that becomes a decreasing fraction of the radial peak value of the standard fully metal-coated tip and deteriorates with respect to the peak value of the $\mathrm{V}$ distribution as well, as illustrated in Figure 16.

The results of the simulations with variable cut height suggest the use of cuts intersecting a larger fraction of the originally axisymmetric probe. As the possible approach to samples with steeper topographic variations would no longer be feasible with cuts at lower heights unless a large cut angle is used at the same time, a trade off with the quality of the achievable $\mathrm{H}$ hot spot is necessary in the choice of the cut height.

The improvement in the peak value of the $\mathrm{H}$ hot spot with steeper cut angles and lower cut heights could be explained as the outcome of a better mode coupling occurring when the asymmetry involves a larger region of the original tip. In fact, in both the cases, a broader area is stripped off by the cut. Such an analysis agrees with what previously observed for other modified structures, ${ }^{18}$ for which asymmetries over more extended portions of the original tip gave rise to an increased coupling between one linearly polarized mode and the radially polarized one. Moreover, in the case of steeper cut angles, the transition from the original taper angle and the angle imposed by the cut is smoother, not so abrupt as is the case for small cut 

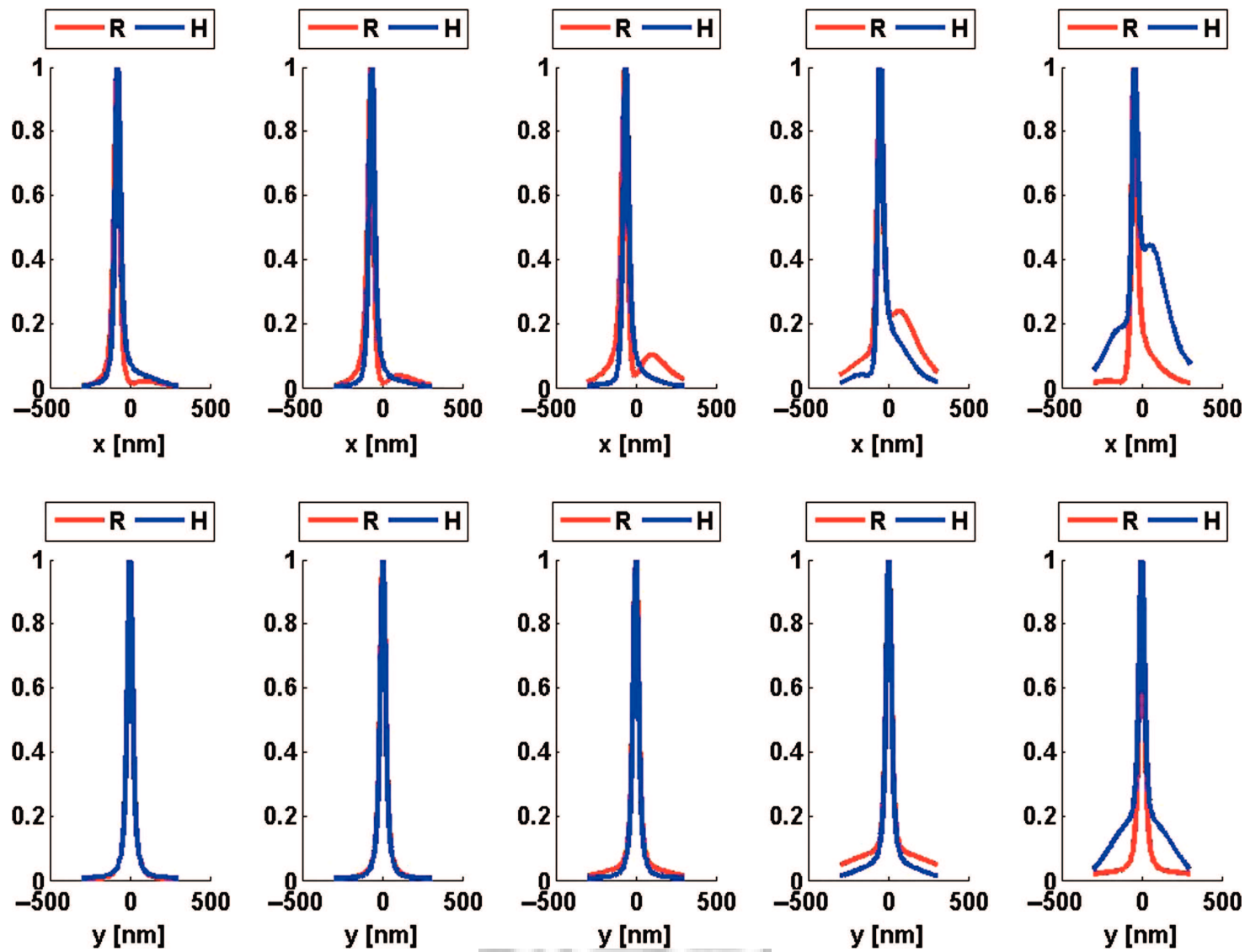

Fig. 13. Normalized cross section profiles for cut probes similar to the one shown in Figure 5 at different cut heights (from left to right the cut height is increased from $741 \mathrm{~nm}$ to $841 \mathrm{~nm}$ with a step of $25 \mathrm{~nm}$; the cut angle is set to $20^{\circ}$ ).

angles. All these considerations justify the highest peak values achieved in these conditions.

As to the practical problem of how to externally excite linearly and radially polarized modes, the procedure described in Refs. [13, 19] could be followed. As uncontrolled injection conditions yield simultaneous excitation of several modes, selective mode coupling based on the injection of optical fields having a spatial distribution similar to the calculated eigenmodes at the tip entrance is necessary. The $\mathrm{H}$ mode can be excited by injecting a $x$-linearly polarized mode, while the $\mathrm{R}$ mode can be excited using

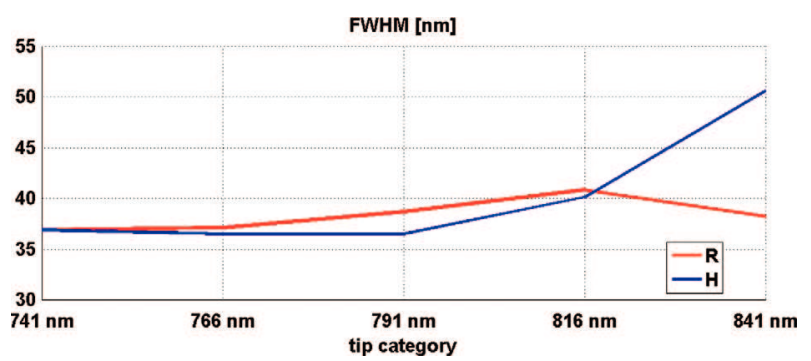

Fig. 14. FWHM for cut probes similar to the one shown in Figure 5 at different cut heights (from left to right the cut height is increased from $741 \mathrm{~nm}$ to $841 \mathrm{~nm}$ with a step of $25 \mathrm{~nm}$; the cut angle is set to $20^{\circ}$ ). liquid crystal elements to generate the radially polarized beam. The quality of the generated beams has to be verified before injecting light in the probe. Good selective coupling into the desired eigenmodes has been shown in the case of the microfabricated cantilever probes reported in the mentioned papers.

Unavoidable structural defects occurring during fabrication such as bubbles or surface irregularities are not expected to change significantly the behaviour observed in the idealized simulated model as long as they are only perturbative and not catastrophic. In fact, as observed in

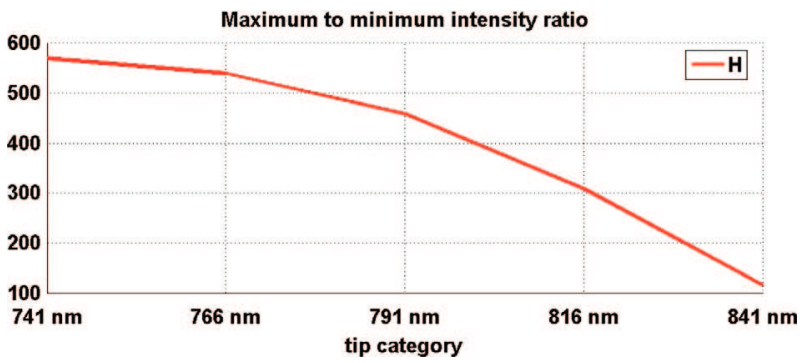

Fig. 15. Contrast for cut probes similar to the one shown in Figure 5 at different cut heights (from left to right the cut height is increased from $741 \mathrm{~nm}$ to $841 \mathrm{~nm}$ with a step of $25 \mathrm{~nm}$; the cut angle is set to $20^{\circ}$ ). 

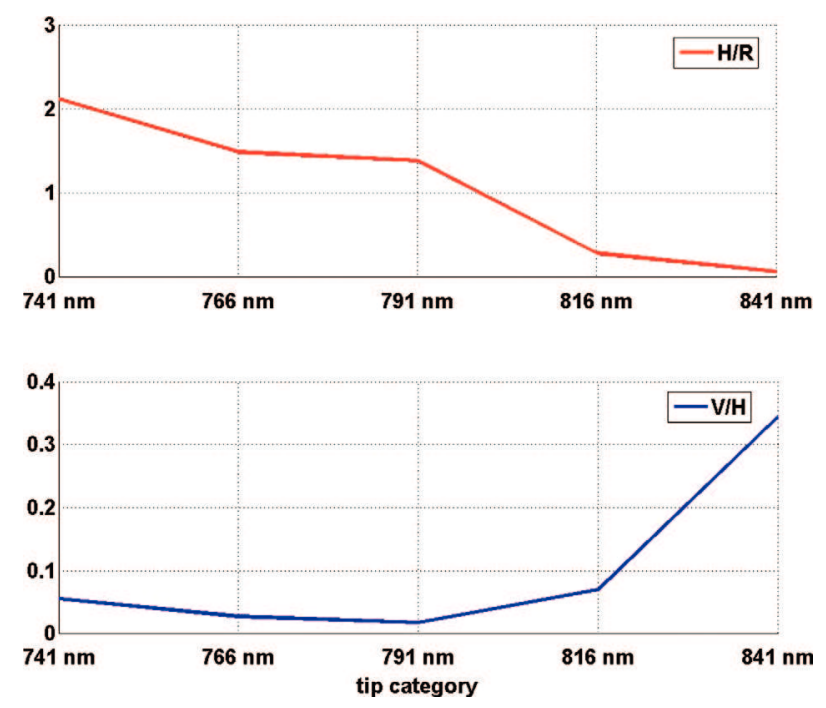

Fig. 16. Ratio between maxima for cut probes similar to the one shown in Figure 5 at different cut heights: the ratio with respect to the $\mathrm{R}$ mode is referred to the radial mode of the fully metal-coated tip (from left to right the cut height is increased from $741 \mathrm{~nm}$ to $841 \mathrm{~nm}$ with a step of $25 \mathrm{~nm}$; the cut angle is set to $20^{\circ}$ ).

Ref. [8], the amount of energy transferred from one mode into another by random perturbative defects is generally small and would not undermine dramatically the characteristics of the field distributions, while the extreme case where defects are so prevalent to perforate significantly the metal coating or degrade the metal layer to the extent that it can no longer be considered as a layer could modify radically the probe behaviour. For example, a big metal bubble close to the tip apex could potentially alter the shape and increase the FWHM of the observed hot spots. ${ }^{20}$ However, this would represent a problem not only for the proposed cut probe, but also for the standard fully metal-coated axisymmetric probe. Fortunately, state of the art fabrication nanotechnologies should allow the production of probes with perturbative, rather than catastrophic defects.

\section{COMBINATION OF ASYMMETRIES}

As already stated, the injection of a radial polarization is quite cumbersome and this has stimulated the search for geometric configurations allowing the coupling of one of the two linearly polarized modes into the radially polarized one. Combination of asymmetries in orthogonal directions can even lead to the coupling of both linearly polarized modes. In this way, any of the three fundamental modes can contribute to the creation of a localized spot. To check the feasibility of this idea, the cut along the $x$ direction has been used in combination with a bilateral slit ${ }^{18}$ parallel to the $x$ axis, thus originating an asymmetry along the $y$ direction. A picture of the analyzed geometry is given in Figure 17.

The height of the cut is $841 \mathrm{~nm}$; its angle amounts to $50^{\circ}$. The slit is $710 \mathrm{~nm}$ deep, $20 \mathrm{~nm}$ wide and offset

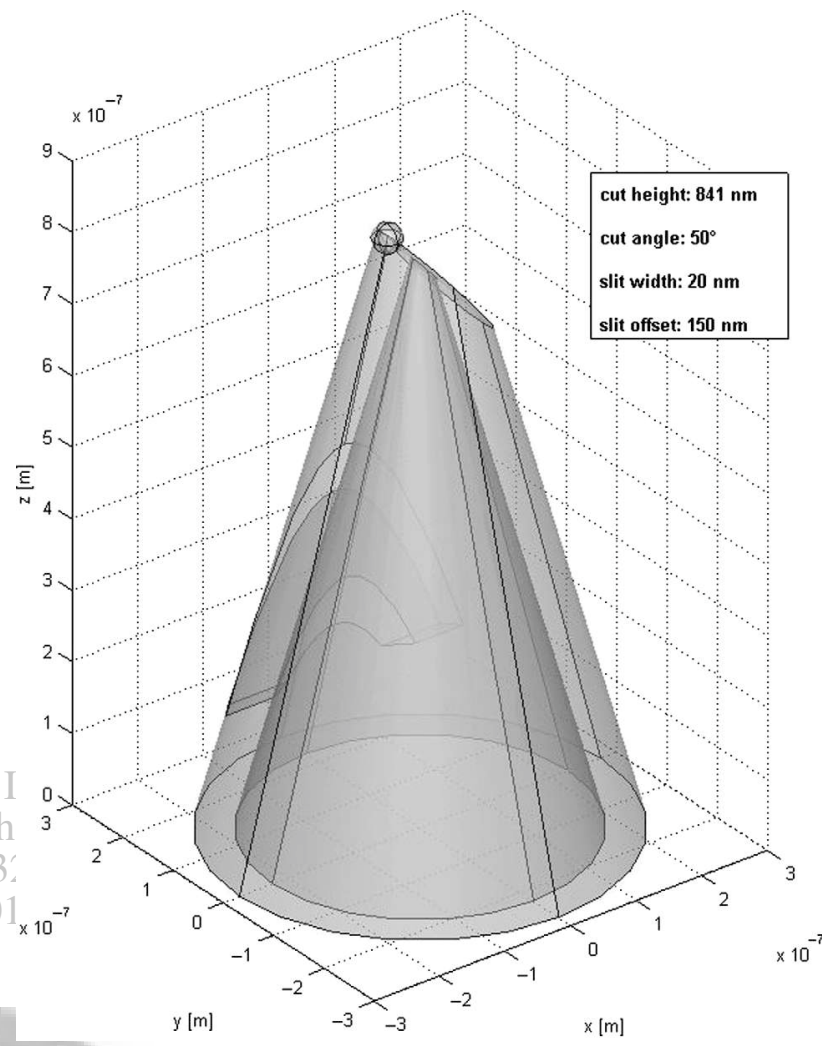

Fig. 17. Probe with orthogonal slit and cut (cut height $841 \mathrm{~nm}$; cut angle $50^{\circ}$; slit depth $710 \mathrm{~nm}$; slit width $20 \mathrm{~nm}$; slit offset $150 \mathrm{~nm}$ ).

$150 \mathrm{~nm}$ from the probe axis. The near field pictures are shown in Figure 18. As expected, the cut leads to field localization for the $\mathrm{H}$ polarized mode, while the slit generates the same effect for the $\mathrm{V}$ mode.

The coupling of both linearly polarized modes had already been considered by Ref. [8] for the case of two identical bubbles in the metal layer at equivalent positions in two orthogonal planes. Of course, the combination of different forms of asymmetries can be considered, for example, two non-intersecting bilateral slits at different heights. The field distributions for such a case are shown in Figure 19. The slit leading to field localization for the $\mathrm{V}$ polarized mode is located at a depth of $872 \mathrm{~nm}$ with an offset of $175 \mathrm{~nm}$, while the slit responsible for the coupling of the $\mathrm{H}$ mode is located at a depth of $572 \mathrm{~nm}$, with an offset of $115 \mathrm{~nm}$. Both slits are $20 \mathrm{~nm}$ wide.

A thorough comparison between these alternative configurations would require a systematic parametric analysis of the influence of different geometric parameters-as done for the single cut configuration - and of the impact of the mutual positions of the orthogonal asymmetries. Note that in the case of the combination of the slit and the cut, the simulated cut was not the best choice in terms of height as suggested by the previous simulations of the single cut. Therefore the localized spot obtained was not optimal neither as to the peak value nor for the FWHM. Hence, these case studies should be regarded as a first 


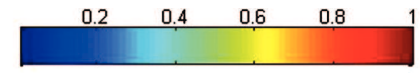

$\mathbf{R}$

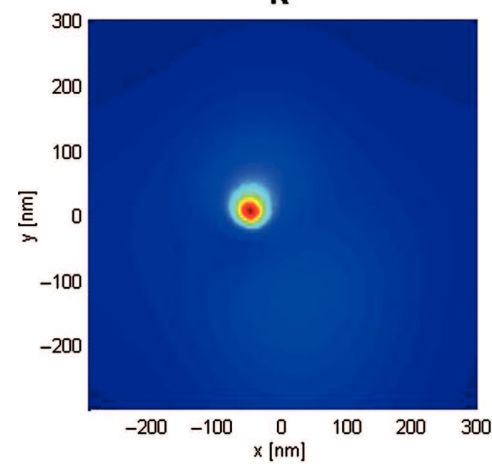

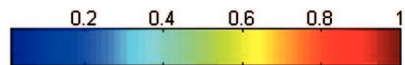

H

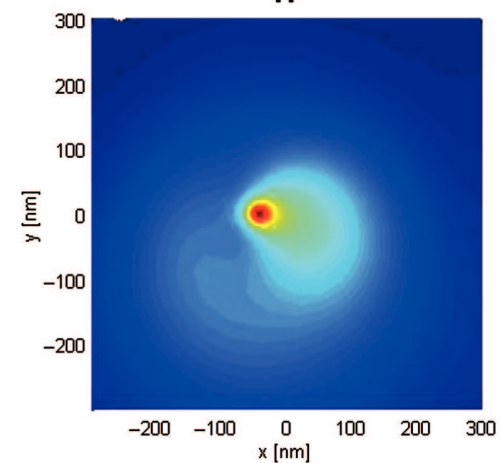

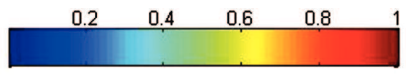

V

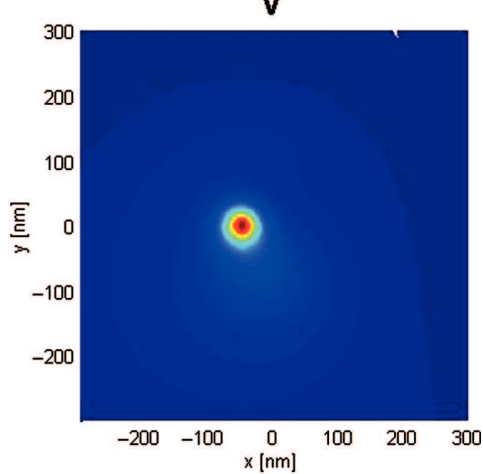

Fig. 18. Normalized electric field intensity at $10 \mathrm{~nm}$ from the apex for the probe with orthogonal slit and cut shown in Figure 17.

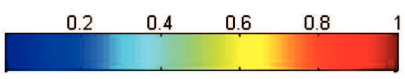

$\mathbf{R}$

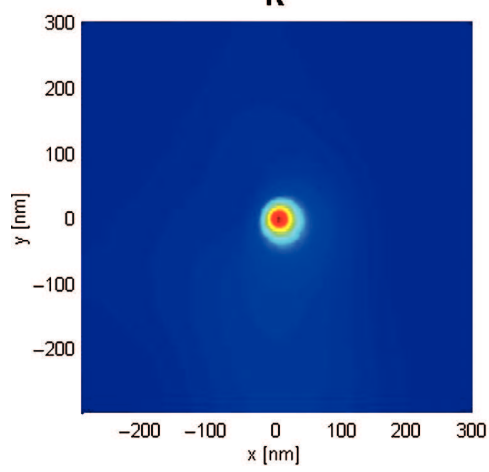

Delivered by Ingenta to:

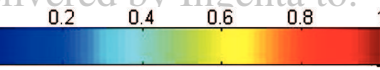

H

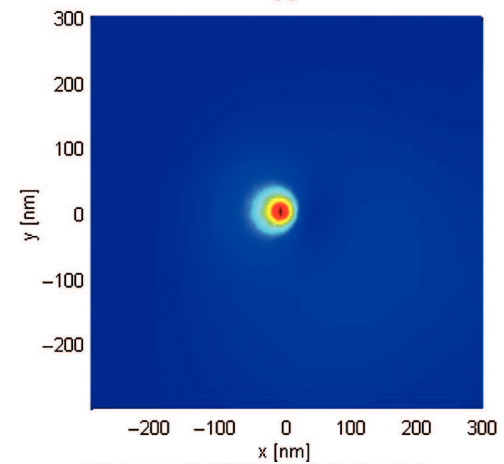

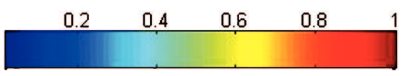

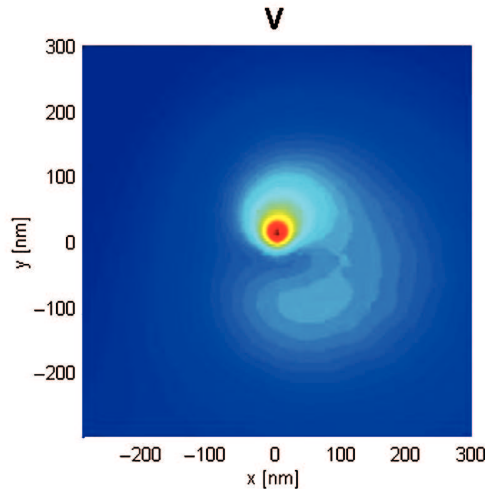

Fig. 19. Normalized electric field intensity at $10 \mathrm{~nm}$ from the apex for the probe with two non-intersecting bilateral slits (first slit: depth $872 \mathrm{~nm}$, offset $175 \mathrm{~nm}$, width $20 \mathrm{~nm}$; second slit: depth $572 \mathrm{~nm}$, offset $115 \mathrm{~nm}$, width $20 \mathrm{~nm}$ ).

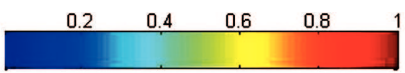

$\mathbf{R}$

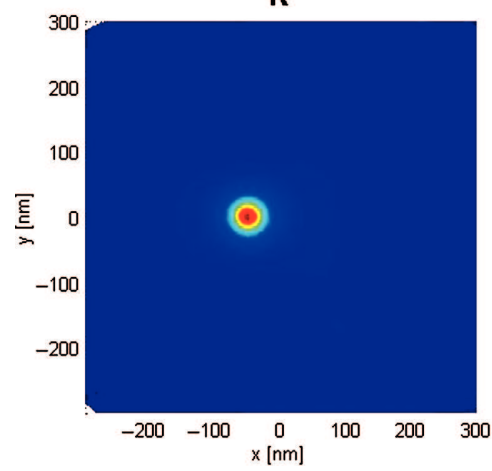

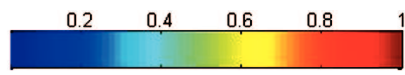

H

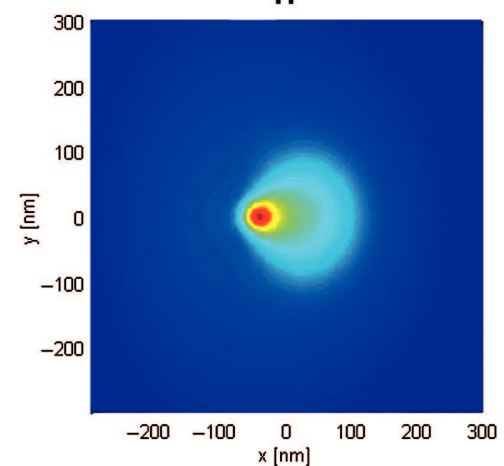

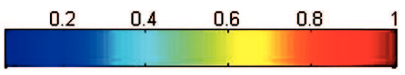

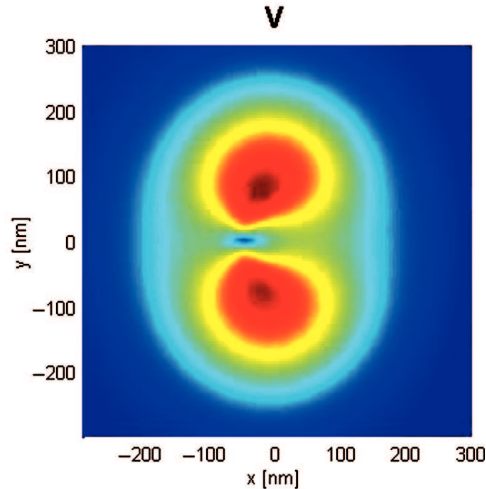

Fig. 20. Normalized electric field intensity at $10 \mathrm{~nm}$ from the apex for the probe with cut and two parallel slits (structure is identical to the one reported in Fig. 17, but with a second slit at a diametrically opposite position). 


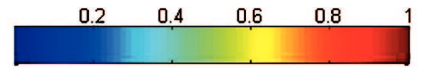

$\mathbf{R}$

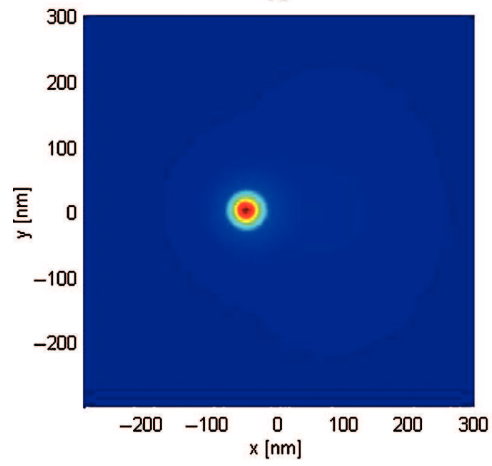

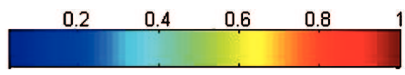

H

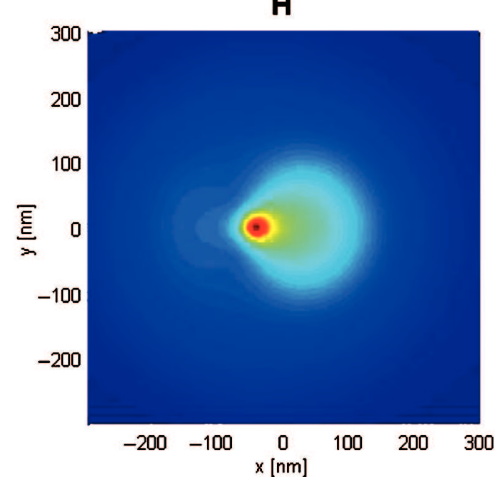

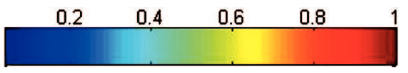

v

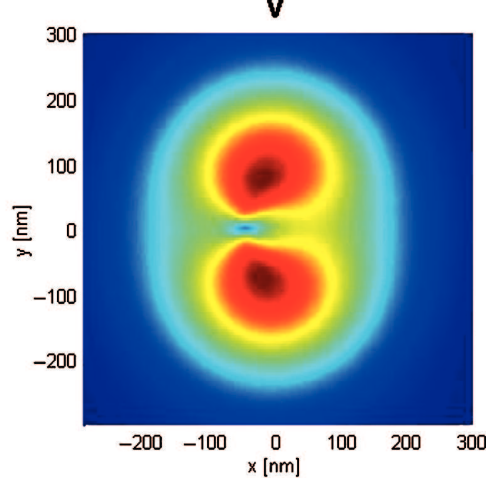

Fig. 21. Normalized electric field intensity at $10 \mathrm{~nm}$ from the apex for the probe with a cut identical to that of Figure 17, but no slits.

qualitative analysis about the feasibility of the combination of the above mentioned asymmetries. A more detailed study would require also a larger computational domain.

A last case was considered to assess the impact of parallel identical modifications in the original geometry. Namely, a probe made up of two identical parallel slits combined with a cut was examined. The structure is identical to the one reported in Figure 17, but with a second slit located at a diametrically opposite position. The resulting field distributions are shown in Figure 20.

For the sake of clarity, the field distributions obtained in the case of a single cut located at the same position are shown in Figure 21. Note that similar results are obtained in both cases. The reason for this is that diametrically opposed modifications lead to destructive interference of the scattering contributions as already suggested in Ref. [8]. Therefore the effects of the single slits cancel each other and no striking difference emerges compared to the case of a single cut without slits.

\section{CONCLUSIONS}

Our simulations show that the proposed form of asymmetry based on an oblique cut is effective in achieving the desired highly localized hot spot by the injection of a linearly polarized input. By cutting the originally fully metal coated SNOM probe along the $x$ direction, strong field localization is realized for the $\mathrm{H}$ polarized mode. The parametric analysis carried out on this configuration and taking into account the height and the angle of the cut showed that steeper angles and lower cuts allow better performances in terms of FWHM and peak value of the $\mathrm{H}$ hot spot. Therefore, the use of this configuration combined with a proper choice of the geometric parameters of the structure would provide the possibility of high resolution SNOM by using a linearly polarized input, thus circumventing the need for the generation of a radially polarized input. Moreover, compared to the slits reported in Ref. [18], the structures proposed in this paper are more practical and easier to be realized with high manufacturing reproducibility.

The second point highlighted in the paper is the feasibility of achieving field localization for both linearly polarized modes by introducing asymmetries in orthogonal directions. Two geometries were studied, the first based on the combination of a cut and a slit and the second resulting from the use of slits in orthogonal directions. Simulations showed that both configurations are promising for this purpose. However, they need to be further scrutinized and optimized by tuning the different geometric parameters that can affect their performance. As highly localized hot spots could be obtained with any of the three fundamental modes, the system would be even more robust against misalignments.

New forms of asymmetries could, in the future, be investigated and compared to the ones already considered. Moreover, experimental verification is needed to validate the results of simulation. Selective coupling of the linearly and radially polarized modes into microfabricated fully metal-coated quartz probes has been demonstrated and near field intensity distributions have been measured experimentally by using a collection SNOM operated in a tip-on-tip configuration, confirming the creation of a highly localized hot spot by the injection of a radially polarized input in a standard probe. ${ }^{13}$ A similar tip-on-tip configuration had already been used to measure the highly efficient excitation of optical near field on an aperture fiber probe with an asymmetric structure. ${ }^{21}$ Such an experimental verification needs to be accomplished also for asymmetric fully metal coated near field probes, which, to the best of our knowledge, have been investigated only numerically up to now. Only the experimental fabrication (for example using focused ion beam to realize the asymmetries) and characterization could reveal whether the investigated structures could be realized with a good level of accuracy with state of the art technology and to which 
extent possible defects intervening in the fabrication steps could influence the final overall performance.

Acknowledgments: The authors gratefully acknowledge the support of the Swiss National Science Foundation.

\section{References}

1. B. Hecht, B. Sick, U. Wild, V. Deckert, R. Zenobi, O. Martin, and D. Pohl, J. Chem. Phys. 112, 7761 (2000).

2. L. Novotny and B. Hecht, Principles of Nano-Optics, Cambridge University Press (2006).

3. L. Novotny, Progress in Optics 50, 137 (2007).

4. D. Pohl, W. Denk, and M. Lanz, Appl. Phys. Lett. 44, 651 (1984).

5. A. Lewis, M. Isaacson, A. Harootunian, and A. Muray, Ultramicroscopy 13, 227 (1984).

6. L. Novotny and C. Hafner, Phys. Rev. E 50, 4094 (1994).

7. L. Liu and S. He, Appl. Opt. 44, 17 (2005).

8. W. Nakagawa, L. Vaccaro, and H. P. Herzig, J. Opt. Soc. Am. A by 23, 1096 (2006).
9. W. Ding, S. R. Andrews, and S. A. Maier, Phys. Rev. A 75, 063822 (2007).

10. H. G. Frey, C. Bolwien, A. Brandenburg, R. Ros, and D. Anselmetti, Nanotechnology 17, 3105 (2006).

11. L. Vaccaro, L. Aeschimann, U. Staufer, H. P. Herzig, and R. Dändliker, Appl. Phys. Lett. 83, 584 (2003).

12. E. Descrovi, L. Vaccaro, L. Aeschimann, W. Nakagawa, U. Staufer, T. Scharf, and H. P. Herzig, Proc. SPIE 5736, 96 (2005).

13. P. Tortora, E. Descrovi, L. Aeschimann, L. Vaccaro, H.-P. Herzig, and R. Dändliker, Ultramicroscopy 107, 158 (2007).

14. N. A. Janunts, K. S. Baghdasaryan, K. V. Nerkarayan, and B. Hecht, Optics Communications 253, 118 (2005).

15. W. Chen and Q. Zhan, Chinese Opt. Lett. 5, 709 (2007).

16. W. Chen and Q. Zhan, Opt. Express 15, 4106 (2007).

17. A. Bouhelier, J. Renger, M. R. Beversluis, and L. Novotny, Journal of Microscopy 210, 220 (2003).

18. W. Nakagawa, L. Vaccaro, H. P. Herzig, and C. Hafner, J. Comput. Theor. Nanosci. 4, 692 (2007).

19. E. Descrovi, L. Vaccaro, L. Aeschimann, W. Nakagawa, U. Staufer, and H. P. Herzig, J. Opt. Soc. Am. A 22, 1432 (2005).

20. O. J. F. Martin and M. Paulus, Journal of Microscopy 205, 147 (2002).

21. T. Yatsui,; M. Kourogi, and M. Ohtsu, Appl. Phys. Lett. 71, 1756 $\underline{(1997)}$

Received: 14 September 2009. Accepted: 28 October 2009. 